\title{
High Seroprevalence of CMV Among Women of Childbearing Age Implicates High Burden of Congenital Cytomegalovirus Infection in Poland
}

\author{
JOANNA SIENNICKA ${ }^{1 *}$, MILENA DUNAL-SZCZEPANIAK ${ }^{1}$, AGNIESZKA TRZCIŃSKA ${ }^{1}$, \\ PAULINA GODZIK ${ }^{1}$ and MAGDALENA ROSIŃSKA ${ }^{2}$
}

\footnotetext{
${ }^{1}$ Department of Virology, National Institute of Public Health - National Institute of Hygiene, Warsaw, Poland ${ }^{2}$ Department of Epidemiology, National Institute of Public Health - National Institute of Hygiene, Warsaw, Poland

Submitted 23 September 2015, revised 29 February 2016, accepted 8 March 2016
}

\begin{abstract}
Cytomegaloviruses are common worldwide, with variable frequency of infections. The infection in pregnancy may lead to pregnancy loss or serious sequelae for the child. To understand the risk posed by CMV in Poland we conducted cross-sectional study on women aged 15-49 basing on existing serum bank. Age dependent CMV incidence, the rates of congenital infection and sequelae were modelled from sero-prevalence, literature and demographic data. The overall anti-CMV IgG prevalence was $81.9 \%$ increasing from $74.3 \%$ in $<30$ years old to $94.3 \%$ in subjects $45+$ years old. The lowest incidence was estimated at the age of 15 and the highest at the age 34 ( 3.8 and 8.95 respectively/100 women/year). The estimated rate of cCMV varies from 22.4 to 37.2 per 1000 live birth depending on the assumptions made. The proportion of cases due to secondary infection ranged from $34.8 \%$ to $49.9 \%$ accordingly.
\end{abstract}

Ke y w ord s: CMV IgG prevalence, CMV in Poland, congenital CMV infection, congenital CMV sequelae, women of childbearing age

\section{Introduction}

Cytomegalovirus (CMV) infections are common with a worldwide distribution. The virus spreads by contact with infected body fluids, usually saliva, urine, blood, or genital secretions (Cannon et al., 2011). After the primary infection (the first infection), despite immune response, CMV establishes a lifelong latency with intermittent reactivations (Sinclair and Sissons, 2006). The infected individual may also experience reinfection by another strain of CMV. During pregnancy, primary or secondary (reactivation or reinfection) maternal infection can lead to transmission through placenta and infecting the fetus, which gives rise to congenital CMV infection (cCMV) (Hyde et al., 2010).

$\mathrm{CMV}$ is the leading cause of congenital infections in humans, resulting in pregnancy loss or disability of the child, including sensorineural hearing loss, developmental delay and mental retardation. The consequences of the infection of a pregnant women depend largely on the type of CMV infection. While primary infection during pregnancy has a higher rate of mother-to-fetus transmission and is more likely to cause symptoms at birth, strong evidence exist that in fact the secondary infections account for the most of the disease burden associated with cCMV (Kenneson and Cannon, 2007; Wang etal., 2011; Yamamoto et al., 2013). The risk of acquiring the primary infection during pregnancy depends both on the incidence and on the proportion susceptible among the pregnant women, which can be approximated by the proportion of women who do not have CMV antibodies. In the situation, in which the majority of pregnant women have already experienced a prior CMV infection the majority of in utero infections will be due to secondary maternal infections. Hence, these infections will become the main source of the CCMV.

Results of the earlier sero-epidemiological studies showed that prevalence of CMV infection depends on age, origin and socioeconomic status. Adults of high socioeconomic status in developed countries may have an antibody prevalence as low as $40 \%$, while in developing countries more than $80 \%$ subjects are CMV positive (Nyholm and Schleiss, 2010). Among women in Europe the seroprevalence level varies from 30\% to $90 \%$ (Ludwig and Hengel, 2009). The last, representative serosurvey in Poland was carried out in 1979 (Imbs and Rudnicka, 1987). Thirty five years ago in Poland,

\footnotetext{
* Corresponding author: J. Siennicka, Department of Virology, National Institute of Public Health - National Institute of Hygiene, Warsaw, Poland; e-mail: jsiennicka@pzh.gov.pl
} 
seroprevalence in women aged 15-39 years old was $83.3 \%$ and these infections are not under surveillance. The burden of congenital disease remains therefore largely unknown and we are currently not able to quantify the risk of infection and its complications to pregnant women and their children. Our aim was to estimate current seroprevalence of CMV among women of the childbearing age in Poland and estimate the risk of $\mathrm{CMV}, \mathrm{cCMV}$ and of the long term sequelae related to $\mathrm{CCMV}$ in order to better inform pregnancy screening and counselling practices as well as other potential strategies to prevent cCMV.

\section{Experimental}

\section{Materials and Methods}

Serum samples. Serum samples for testing were selected from a serum bank collected in 2010-2011 (Project NN404 191636; ethics statements 6/2008 - Committee of Bioethics at NIPH-NIH, 19.08.2008). Serum bank, intended to represent the general population, included 4822 serum samples collected by cluster sampling method, with clusters defined by the hospital unit and specific day within the recruitment period. The selection of 21 hospital units was made randomly from "Register of Healthcare Units" circumscribed to orthopedic and general surgery wards in 5 geographical regions of Poland (lubelskie, mazowieckie, świętokrzyskie, warmińsko-mazurskie and wielkopolskie). Each individual participating in the project was asked to provide written consent to include their anonymized serum sample for future epidemiological investigations of infectious diseases (serological or molecular). Serum samples were collected from all consenting adult patients fulfilling the following criteria: admitted to the hospital because of emergency indications (accidents, injuries) or hospitalized for appendicitis, hernia, or the gallbladder inflammation as described in (Godzik et al., 2012). Sera were aliquoted, catalogued and stored at $-70^{\circ} \mathrm{C}$. The consent forms are stored within the medical records at the laboratory, which performed diagnostic testing for the initial project. The serum bank is maintained by the Department of Virology, National Institute of Public Health - National Institute of Hygiene and is fully anonymous (http://www.pzh.gov. $\mathrm{pl} /$ page/index.php? $\mathrm{id}=1075 \& \mathrm{~L}=1$ ). The bank is accessible for further sero-epidemiological research upon acceptance by the project committee. We studied a total of 712 samples collected from women in childbearing age in the following age groups: <30 years (218), $30-34$ years (105), 35-39 years (133), 40-44 years (114), $45+$ years (142).
Laboratory test and quality control. IgG antibodies specific to cytomegalovirus were detected using an indirect enzyme immunoassay (ETI-CYTOK-G PLUS test, DiaSorin, Italy). The antibody level was expressed in international units per millilitre $(\mathrm{mIU} / \mathrm{ml})$. For the internal quality control purpose the $10 \%$ of tested samples $(n=70)$ were blindly retested with the same test kit. Internal quality control showed $100 \%$ concordant results (61 positive and 9 negative).

Statistical analysis. We used chi-square test and logistic regression to compare the prevalence of CMV across age groups, region and type of residence (urban/ rural). Relation by age was investigated both using age group and with the fractional polynomial model, but for simplicity of interpretation age group is shown in the prevalence model. Likelihood ratio test was used to test the effects of covariates in the logistic model.

Estimation of the force of infection and incidence. Assuming life-long immunity (demonstrated by detectable antibodies), negligible mortality due to the CMV infection and stability of the force of infection over time we were able to estimate the age dependent force of infection $l(a)$ based on the serology results using the catalytic model:

$$
\frac{d}{d a} q(a)=-l(a) \times q(a),
$$

Where $q(a)=1-\pi(a)$ is the proportion of susceptible individuals among the population of age $a, \pi(a)$ is prevalence of antibodies at the age a, so $q(a)=$ $=e^{-\int_{0}^{a} l(s) d s}$

The force of infection, $l(a)$, can be derived as follows:

$$
l(a)=\frac{\pi^{\prime}(a)}{1-\pi(a)}
$$

The prevalence is modeled with a linear model with link function $g$ and the linear predictor $\eta(a)$ :

$$
\pi(a)=g^{-1}(\eta(a))
$$

In order to ensure a flexible choice of the model for the force of infection we used the fractional polynomial model for $\eta(a)$ with logit and complementary log-log link functions $g$ to model the age dependent prevalence $\pi$ (a) (Shkedy et al., 2006).

For the logit link function we have the following equality for the force of infection:

$$
l(a)=\eta^{\prime}(a) \times \pi(a)
$$

We selected best fitting models based on deviance criterion and Akaike information criteria (AIC) for selecting among models with different link function. We restricted the considered models to those estimating positive force of infection. Based on these criteria we selected logistic model with

$$
\eta(a)=\beta_{0}+\beta_{1} \times a^{2}+\beta_{2}+a^{2} \times \ln (a) .
$$


Substituting the formula for $\eta(a)$ the force of infection is estimated according to the formula:

$$
l(a)=\left(2 \times \beta_{1}+\beta_{2} \times a \times[2 \times \ln (a)+1] \times \pi(a)\right.
$$

The estimated force of infection describes the rate of infections among the susceptible fraction of the population. In order to inform the burden of disease among women of the childbearing age and the possible impact on congenital infections we estimate the rate of infections in the entire population, incidence $I(a)=l(a) \times q(a)$.

Estimation of the burden of congenital CMV infection. We estimated the rate of congenital infection per 1000 births assuming:

- the incidence in pregnancy is not different then among non-pregnant women of the same age and the childbearing age is restricted to $15-49$ years

- the rate of transmission to fetus in case of previously seronegative pregnant women is $32 \%$ (Kenneson and Cannon, 2007)

- the rate of cCMV due to primary maternal infections, $R_{\text {prim }}$ will be estimated by summing mother age specific rates (child bearing age):

$$
\begin{aligned}
& R_{\text {prim }}=\frac{1}{\mathrm{~B}} \times T_{1} \times \sum_{\mathrm{a}=15}^{49} D \times l(a) \times q(a) \times B(a) \\
& B=\sum_{\mathrm{a}=15}^{49} B(a)
\end{aligned}
$$

Where $B(a)$ number of births given by mothers aged a, $B$ is the total number of life births $D$ is the time at risk for infection resulting in $\mathrm{cCMV}$, as fraction of year and $T_{1}$ the transmission rate to fetus, $T_{1}=32 \%$. The calculations based on the number of births (live and dead) registered in 2011, according to the maternal age, $B(a)$ (Central Statistical Office of Poland).

- the rate of cCMV due to secondary infections, $R_{\text {sec }}$, is estimated in two different scenarios:

- "reinfection model": assuming that the reinfections occur at the same rate as primary infections (force of infection $l(a)$ ), cCMV is associated only with reinfection and the transmission rate to fetus, $T_{2}$, is related to $T_{1}$ as followed: $T_{2}=0,31 \times T_{1}$. The cCMV rate per 1000 live births in the "reinfection model", $R_{\text {sec }}=R_{\text {reinf }}$ is:

$$
\begin{aligned}
& R_{\text {reinf }}=\frac{1}{\mathrm{~B}} \times \sum_{\mathrm{a}=15}^{49} D \times T_{2} \times l(a) \times(1-q(a)) \times B(a)= \\
& =\frac{1}{\mathrm{~B}} \times \sum_{\mathrm{a}=15}^{49} D \times 0.31 \times T_{1}(a) \times(1-q(a)) \times B(a)
\end{aligned}
$$

- "reactivation model": the cCMV is associated only with reactivation, i.e. not related to the reinfection rate. We further assume that every seropositive woman has the same chance of transmitting the virus to the fetus independently of the incidence of CMV in the population. In particular this proportion will not vary by age. The rate of CCMV, $R_{\text {sec }}=R_{\text {react }}$ inputted in this model is based on the proportion observed in other countries as proposed by de Vries et al. (2013):

$$
R_{\text {react }}=\frac{P}{1+\exp (6.15-2.44 \times P)}
$$

where $P$ is the seroprevalence among pregnant women estimated based on seroprevalence among women of childbearing age weighted by the number of birth by maternal age:

$$
P=\frac{1}{\mathrm{~B}} \times \sum_{a=15}^{49}(1-q(a)) \times B(a)
$$

- the proportion of cCMV infections that are symptomatic infections at birth is $S_{1}=15 \%$ for cCMV related to primary infection and $S_{2}=1 \%$ for those related to secondary infection (Gardella, 2008). The rate of cCMV symptomatic at birth per 1000 birth, $R_{\text {sympt }}$ was evaluated as:

$$
R_{\text {sympt }}=R_{\text {prim }} \times S_{1}+R_{\text {sec }} \times S_{2}
$$

- the proportion of long term sequelae is $L_{1}=90 \%$ for children with cCMV symptomatic at birth and $\mathrm{L}_{2}=10 \%$ for those without symptoms at birth (Gardella, 2008). The rate of long term sequelae, $R_{\text {seq }}$, was calculated as:

$$
R_{\text {seq }}=R_{\text {sympt }} \times L_{1}+\left(R_{\text {prim }}+R_{\text {sec }}-R_{\text {sympt }} \times L_{2}\right)
$$

We considered the following scenarios:

Scenario 1 (no pregnancy loss/reinfection): there is no pregnancy loss associated with CMV infection and the cCMV due to secondary infection is related to reinfections.

Scenario 2 (first trimester pregnancy loss/reinfection): all infections in the first trimester lead to pregnancy loss and thus only the infections in second and third trimester lead to cCMV, and the cCMV due to secondary infection is related to reinfections.

Scenario 3 (no pregnancy loss/reactivation): there is no pregnancy loss associated with CMV infection and cCMV associated with secondary infections is related to reactivations.

Scenario 4 (first trimester pregnancy loss/reactivation): all infections in the first trimester lead to pregnancy loss and cCMV associated with secondary infections is related to reactivations.

For the four scenarios we evaluated the overall cCMV rate, the rate of symptomatic infections at birth and the rate of long term sequelae. The calculations based on the number of births (live and dead) registered in 2011, according to the maternal age (Central Statistical Office of Poland).

The confidence intervals for the force of infection, the cCMV rates, the rates of symptomatic infections and 
Table I

Factors independently associated with higher anti-CMV prevalence among women of child-bearing age in Poland.

\begin{tabular}{|l|l|c|c|c|c|c|}
\hline \multicolumn{2}{|l|}{} & $\begin{array}{c}\text { No. positive/ } \\
\text { No. tested }\end{array}$ & $\begin{array}{c}\text { Prevalence } \\
(\%)\end{array}$ & $\begin{array}{c}\text { Adjusted } \\
\text { odds ratio }\end{array}$ & 95\% C I & $\begin{array}{c}\text { p-value } \\
\text { (LR test) }\end{array}$ \\
\hline Age group (years) & $<30$ & $162 / 218$ & 74.3 & Reference & & \\
\hline & $30-34$ & $76 / 105$ & 72.4 & 0.96 & $0.57-1.64$ & $<0.0001$ \\
\hline & $35-39$ & $111 / 133$ & 83.5 & 1.83 & $1.05-3.19$ & \\
\hline & $40-44$ & $100 / 114$ & 87.7 & 2.54 & $1.34-4.81$ & \\
\hline & $45+$ & $134 / 142$ & 94.4 & 5.91 & $2.71-12.89$ & \\
\hline Residence & Rural & $265 / 323$ & 82.0 & Reference & & \\
\hline & Urban & $317 / 388$ & 81.7 & 0.92 & $0.62-1.37$ & 0.674 \\
\hline & Wielkopolskie & $137 / 177$ & 77.4 & Ref. & & 0.2180 \\
\hline & Lubelskie & $98 / 122$ & 80.3 & 1.35 & $0.75-2.43$ & \\
\hline & Mazowieckie & $163 / 200$ & 81.5 & 1.38 & $0.82-2.31$ & \\
\hline & Swiętokrzyskie & $101 / 116$ & 87.1 & 1.99 & $1.02-3.86$ & \\
\hline & Warmińsko-mazurskie & $84 / 97$ & 86.6 & 1.89 & $0.94-3.81$ & \\
\hline
\end{tabular}

long term sequalae were estimated by parametric bootstrap method resampling 1000 times (Normal-based bootstrap confidence intervals, BCI). In this method the model for prevalence was assumed to be correct, the mean adjusted standardized Pearson's residuals were resampled and combined with the model estimated values to create the bootstrapped dataset (Davidson and Hinkley, 1997). Statistical analysis was carried out in STATA version 13.1. The confidence intervals were calculated only taking into consideration variability arising from seroprevalence data with all the literature parameters assumed to be known.

\section{Results}

CMV IgG prevalence and estimated incidence of primary CMV infection among women of childbearing age. The overall prevalence of CMV IgG among women of childbearing age was $81.9 \%$ (583 out of 712 ). The number of negative samples was 129 (18.1\%). There were no equivocal results. The prevalence was age-dependent and increasing from $74.3 \%$ in $<30$ years old to $94.3 \%$ in subjects $45+$ years old.

In multivariable analysis age was the most important predictive factor for CMV prevalence (Table I). Additionally we note regional differences, although the overall contribution of the factor on likelihood ratio test is insignificant. On the other hand the difference between the rural and urban residence was small and insignificant.

Observed and predicted prevalence by age was shown on Fig. 1. It should be noted that for the age range in question there is an almost linear increase.

Figure 2 depicts the incidence of primary infection in the population of the observed level of susceptibil- ity. The lowest incidence is expected at the age of 15 (3.8 per 100 women per year) and the highest during the age $30-40$ (the maximum 8.95 at the age 34 ). For higher ages the incidence then declines due to universal immunity, although the force of infection remains high (data not shown). The estimate of the overall incidence among women of childbearing age is 6.98 per 100 per year (Normal-based 95\% Bootstrap CI, BCI, 5.15-8.0).

Estimated burden of congenital CMV infections in Poland. CMV seroprevalence standardized to the age distribution of the pregnant women in 2011 was $75.3 \%$ (95\% CI $70.7-79.4 \%$ ) and the estimated incidence of primary infection in this group - 7.76 per 100 per year (95\% BCI 5.68-9.85). The estimated rate of cCMV varies from 22.4 to 37.2 per 1000 live birth depending on the scenario, with non-overlapping $95 \%$ confidence intervals for the highest and the lowest esti-

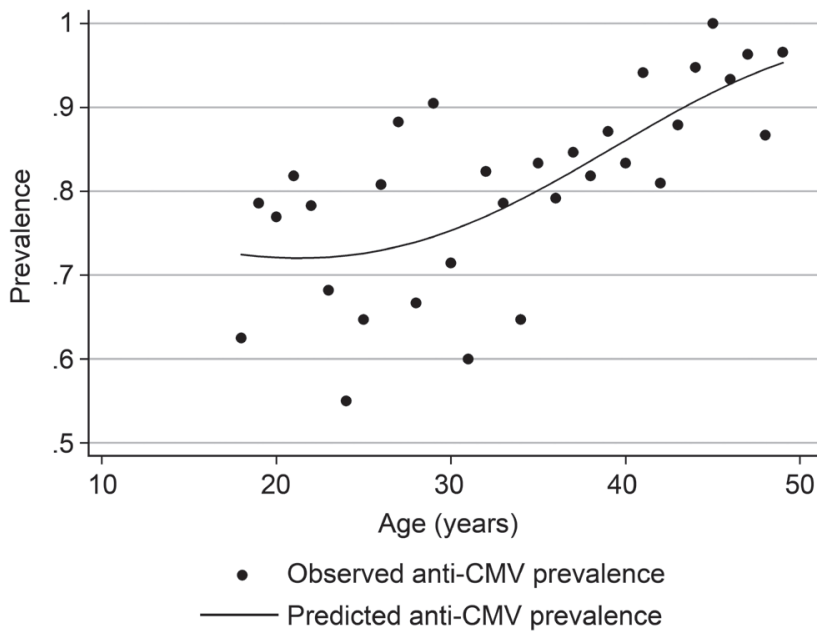

Fig. 1. Prevalence of CMV IgG among women of childbearing age. Predicted prevalence is based on the accepted fractional polynomial model relating the prevalence to the woman's age. 


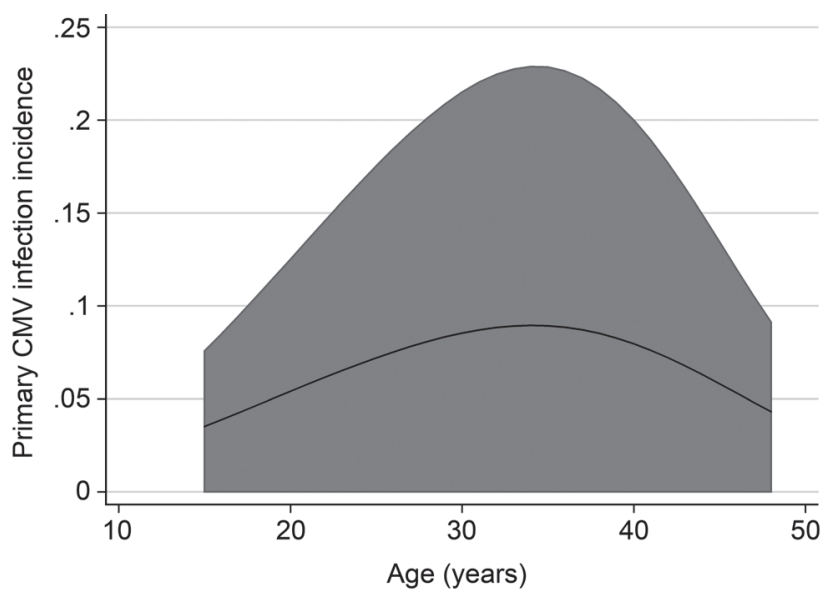

Fig. 2. Estimated annual incidence of primary CMV infection per 100 women of childbearing age.

The incidence is estimated from observed prevalence based on catalytic model for the force of infection combined with fractional polynomial prevalence model. The $95 \%$ confidence band (shaded area) is obtained through parametric bootstrap method. mate (Table II). The difference was especially apparent for older ages of the mother (Fig. 3). These estimates included infections due to primary infection of the pregnant mother at 18.6 per 1000 live births in scenarios 1 and 3, assuming no relation of CMV infection to pregnancy loss, or 12.4 in scenarios 2 and 4 assuming all infections in the first trimester lead to pregnancy loss. The estimates of the number of cCMV rates due to secondary infections were higher in the scenarios 1 and 2, relating the risk of cCMV to the force of infection, which may be viewed as re-infection model. The proportion of cases due to secondary infection ranged from $34.8 \%$ to $49.9 \%$ accordingly (Table II).

The rate of infections symptomatic at birth ranged across the scenarios from 1.9 to 3 per 1000 births, for all of the scenarios being the highest among women aged 30-35. The rates of long-term sequelae had a similar peak for the scenarios 3 and 4 , but for the scenarios 1 and 2 were increasing for older ages of the mother (Fig. 3).
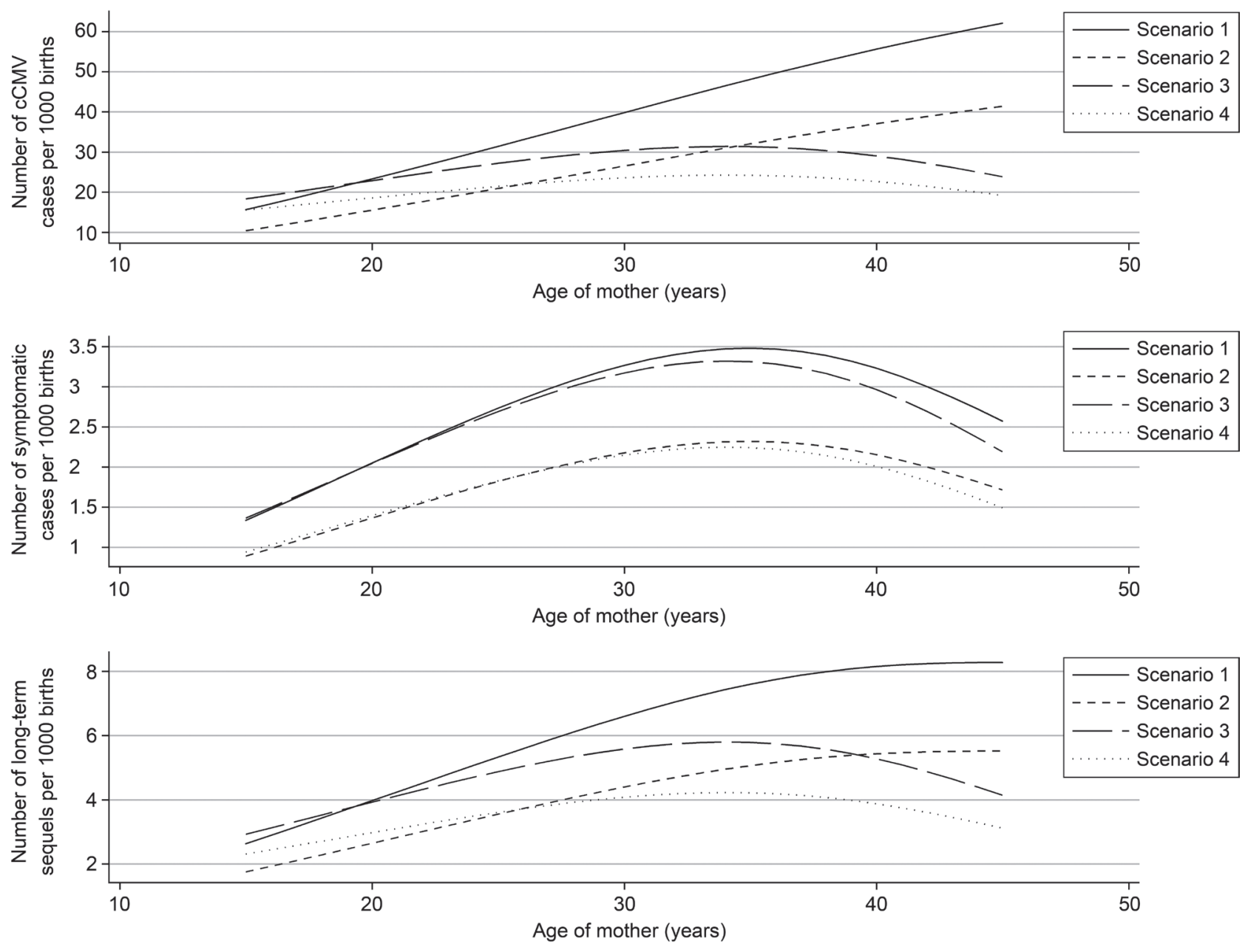

Fig. 3. Predicted rate of cCMV, cCMV symptomatic at birth and long-term sequelae, by mother's age.

The rates of $\mathrm{CCMV}$, cCMV symptomatic at birth and the long term sequelae are estimated from estimated force of infection, estimated prevalence and published values of individual risk for symptoms at birth and development of long term sequelae. The following scenarios were used: Scenario 1. No pregnancy loss due to CMV infection/ cCMV due to secondary infection related to reinfections; Scenario 2. All infections in the first trimester lead to pregnancy loss/ cCMV due to secondary infection is related to reinfections; Scenario 3. No pregnancy loss due to CMV infection/ cCMV due to secondary infections related to reactivations; Scenario 4 . All infections in the first trimester lead to pregnancy loss/ cCMV due to secondary infections related to reactivations. 
Table II

Estimated rates of congenital (symptomatic and asymptomatic) CMV infection (cCMV) and frequency of long term sequels.

\begin{tabular}{|c|c|c|c|c|c|}
\hline \multirow{2}{*}{\multicolumn{2}{|c|}{ CMV infection sequelae }} & \multicolumn{4}{|c|}{ Observed estimate (normal-based 95\% Bootstrap CI) } \\
\hline & & \multirow{2}{*}{$\begin{array}{c}\text { Scenario } 1 \\
14520 \\
(10766-18274)\end{array}$} & \multirow{2}{*}{$\begin{array}{c}\text { Scenario } 2 \\
9680 \\
(7177-12182)\end{array}$} & \multirow{2}{*}{$\begin{array}{c}\text { Scenario } 3 \\
11155 \\
(9229-13081)\end{array}$} & \multirow{2}{*}{$\begin{array}{c}\text { Scenario } 4 \\
8732 \\
(7456-10008)\end{array}$} \\
\hline cCMV cases & Total number & & & & \\
\hline & Rate per 1000 birth & $\begin{array}{c}37.2 \\
(27.6-46.8)\end{array}$ & $\begin{array}{c}24.8 \\
(18.4-31.2)\end{array}$ & $\begin{array}{c}28.6 \\
(23.7-33.5)\end{array}$ & $\begin{array}{c}22.4 \\
(19.1-25.7)\end{array}$ \\
\hline \multirow[t]{2}{*}{ cCMV cases symptomatic at birth } & Total number & $\begin{array}{c}1163 \\
(852-1474)\end{array}$ & $\begin{array}{c}775 \\
(568-983)\end{array}$ & $\begin{array}{c}1129 \\
(836-1422)\end{array}$ & $\begin{array}{c}766 \\
(571-961)\end{array}$ \\
\hline & Rate per 1000 birth & $\begin{array}{c}3 \\
(2.2-3,8)\end{array}$ & $\begin{array}{c}1.9 \\
(1.4-2.4)\end{array}$ & $\begin{array}{c}2.9 \\
(2.1-3.6)\end{array}$ & $\begin{array}{c}2 \\
(1.5-2.5)\end{array}$ \\
\hline \multirow[t]{2}{*}{$\begin{array}{l}\text { cCMV cases with expected long } \\
\text { term sequel }\end{array}$} & Total number & $\begin{array}{c}2382 \\
(1758-3006)\end{array}$ & $\begin{array}{c}1588 \\
(1172-2004)\end{array}$ & $\begin{array}{c}2019 \\
(1592-2445)\end{array}$ & $\begin{array}{c}1486 \\
(1202-1769)\end{array}$ \\
\hline & Rate per 1000 birth & $\begin{array}{c}6.1 \\
(4.5-7.7)\end{array}$ & $\begin{array}{c}4.1 \\
(3-5.1)\end{array}$ & $\begin{array}{c}5.2 \\
(4.1-6.3)\end{array}$ & $\begin{array}{c}3.8 \\
(3.1-4.5)\end{array}$ \\
\hline \multirow[t]{2}{*}{$\begin{array}{l}\text { cCMV associated with secondary } \\
\text { infection in pregnancy }\end{array}$} & Total number & $\begin{array}{c}7251.8 \\
(5449.3-9054.3)\end{array}$ & $\begin{array}{c}4834.6 \\
(3632.9-6036.2)\end{array}$ & $\begin{array}{c}3886.7 \\
(3806.1-3967.4)\end{array}$ & $\begin{array}{c}3886.7 \\
(3806.1-3967.4)\end{array}$ \\
\hline & Proportion (\%) & $\begin{array}{c}49.9 \\
(49.2-50.7)\end{array}$ & $\begin{array}{c}49.9 \\
(49.2-50.7)\end{array}$ & $\begin{array}{c}34.8 \\
(23.8-45.9)\end{array}$ & $\begin{array}{c}44.5 \\
(33.7-55.3)\end{array}$ \\
\hline
\end{tabular}

\section{Discussion}

We estimate that $81.9 \%$ of women of childbearing age in Poland have been infected with CMV, as evidenced by presence of CMV IgG antibodies. The sero-prevalence increased from $74.3 \%$ in $<30$ years old to $94.3 \%$ in subjects $45+$ years old, suggesting the decreasing with age pool of women susceptible to primary infection. Accounting for the distribution of the births by mother's age we were able to produce overall estimates of congenital infection burden. Assuming no CMV related pregnancy loss, the rate of cCMV was estimated at 37.2 (95\% CI 27.6-46.8) per 1000 births under re-infection model or 28.6 (95\% CI 23.7-33.5) under re-activation model. Conversely, if all women infected in the first trimester of pregnancy miscarried, the rates of cCMV would be respectively 24.8 (95\% CI 18.4-31.2) and 22.4 (95\% CI 19.1-25.7) per 1000 births.

The results of our study showed that seroprevalence in Poland is higher than in the majority western European countries in white women with: $30.4 \%$ in Ireland within Irish women (Knowles et al., 2005), 45,6\% in The Netherlands (Kordenwal et al., 2015), 45.9\% in United Kingdom within white women (Tookey et al., 1992), $47.5 \%$ in Germany (Enders et al., 2003), 51.5\% in France (Gratacap-Cavallier et al., 1998), 57.4\% in Spain (de Ory etal., 2004), 70.7\% in Finland (Mustakangas et al., 2000), 72\% in Sweden (Engman et al., 2008). It was also observed that seroprevalence in 2010 (81.9\%) not much differ from this observed in 1979 (Imbs and Rudnicka, 1987) (83.3\%). High prevalence, which is comparable to the estimates from before 35 years as well as little regional differences suggest persistent and universal existence of factors driving CMV transmission in Poland. These factors have not been well studied, but taking into account significant burden of disease more attention should be given to establish the contexts of transmission.

In line with the difference in seroprevalence between women in childbearing age in Poland and in other developed European countries, the rate of cCMV estimated in our model, 22.4 up to 37.2 per 1000 births depending on assumed scenario, is higher from the results of two independent meta-analyses performed by Kenneson et al. (2007) and Dollard et al. (2007) - 0.64\% and $0.7 \%$ accordingly. Numerous previous studies noted the positive correlation between the seroprevalence among pregnant women and the rates of cCMV (Kenneson and Cannon, 2007; Ludwig and Hengel, 2009; de Vries et al., 2013). This is explained by the fact that the higher seroprevalence indicates higher force of infection and therefore a higher risk of primary infection in seronegative pregnant women, but also the higher risk of reinfection among the seropositive women.

Incidence estimated based on the seroprevalence cross-sectional sample is low among women below 20 and peaks at the age of 30-35 (Fig. 2). Women in their late 30-ties or early 40-ties are more likely to have contact with small children of their own or in their surroundings and may be therefore exposed to CMV. This is compatible with the observation that having a child was a risk factor for infection (Kiss at al., 2002; Revello et al., 2011). The virus activation from latency is the multifactorial process in which the stress affecting the immunological system could trigger reactivation (Liu et al., 2010). The significant proportion 
of burden of cCMV illness was also attributed to secondary infection of pregnant women by other authors (Wang et al., 2011). In the developed countries this proportion was even higher than in our study, possibly in relation to the lower force of infection and thus lower rate of primary infections.

Our results, especially if considered by age, were sensitive to the assumptions regarding the role of reinfection/reactivation. While it is known that the role of non-primary maternal infections as a source of congenital infection is very important, it is not known what extent of cCMV are caused by viral reactivation versus reinfection with a different viral strain. Observed epidemiological patterns such as the difference between the rate of cCMV in infants born to low income women ( 1 to 2 percent) and the rate in those borne to middle or high income women ( 0.1 to 0.2 percent) cannot be explained solely by reactivation (Boppana et al., 2001). Accordingly it was suggested that reinfection plays a crucial role in cCMV development. Further, some studies have demonstrated association of the reactivity to new CMV strains in mothers who were initially seropositive with cCMV (Boppana et al., 2001; Yamamoto et al., 2010; Ikuta et al., 2013). However, our "re-infection model" produces higher overall estimates than "re-activation model", for which the overall estimates rely on frequencies observed in other countries. We therefore conclude that although the age patterns seen in the "re-infection" models are more plausible then those in "re-activation" model the "re-infection" models may in fact overestimate the true number of cCMV. Mixed acquisition models for secondary infection could be also considered. However, at this point without additional data to inform the "mixture" parameters we would not be able to judge whether these models provide more realistic estimated. We therefore presented only the "extreme" models.

Attribution of cCMV associated with secondary infection to re-infection has implications for public health interventions aiming at behavioral change. As the children are the main source of virus and then could be a cause of re-infection in their mothers, avoiding exposure to CMV through behavioral changes, mainly increasing hygiene, should be beneficial for seropositive as well as seronegative pregnant women (Kiss et al., 2002; Revello et al., 2008; Price et al., 2014).

We also observed, that for mothers approaching 40 or older even over $60 \%$ of long term sequelae may develop despite the lack of symptoms at birth. This estimate is supported by other studies indicating that the majority of children with cCMV infection who will developed subsequent late-onset hearing loss at the first 6 years of life have a normal hearing at birth and could be missed in newborn hearing screening (Fowler et al., 1999). Therefore, to detect the late onset and progression of hearing loss the continued monitor- ing in population of cCMV infected children should be undertaken (Dahle et al., 2000). Similarly as in case of hearing loss, the role of asymptomatic infection at birth may be underestimated in case of other cCMV sequelae as vision impairment and motor/cognitive deficit (Manicklal et al., 2013). Given the high birth prevalence of cCMV in Poland the universal screening for cCMV should be considered.

The limitations of our study include that not much data on the important model parameters such as proportion of symptomatic infections and the proportion of long term sequelae are available. Simple numbers available from literature were used for the estimations in this paper, which overestimates our confidence in the results. No reliable information could be found of the rates of pregnancy loss in case of maternal infection and the differentiation between the role of reinfection and reactivation is still not well studied. We therefore considered four alternative scenarios and showed that the results are sensitive to the assumptions made. This allowed us to understand the possible ranges of cCMV rate, although we were not able to produce a single number for cCMV rate estimation.

\section{Conclusion}

We demonstrated persistently high burden of cCMV in Poland, higher than in neighboring countries. The reason for this difference is unknown, which should stimulate further research to clarify the level of awareness of the health providers and the pregnant women of the infection (Adler et al., 2004; Revello et al., 2011; Cordier et al., 2012a; 2012b) and inform the public health interventions. While the CMV IgG negative pregnant women should be still counselled about the risk of acquiring CMV, the importance of secondary infections in pregnancy, despite the low risk of it affecting the child on individual level, should be emphasised. Taken into the consideration the role and the impact of secondary infections, the prevention measures, such as vaccine or behavioral interventions, will be potentially beneficial not only to prevent primary infections but also secondary one.

\section{Literature}

Adler S.P., J.W. Finney, A.M. Manganello and A.M. Best. 2004. Prevention of child-to-mother transmission of cytomegalovirus among pregnant women. J. Pediatr. 145: 485-491.

Boppana S.B., L.B. Rivera, K.B. Fowler, M. Mach and W.J. Britt. 2001. Intrauterine transmission of cytomegalovirus to infants of women with preconceptional immunity. N. Engl. J. Med. 344: 1366-1371.

Cannon M.J., T.B. Hyde and D.S. Schmid. 2011. Review of cytomegalovirus shedding in bodily fluids and relevance to congenital cytomegalovirus infection. Rev. Med. Virol. 21: 240-255. 
Central Statistical Office of Poland (CSOP). 2014. http://stat.gov. $\mathrm{pl} / \mathrm{en} /, 2$ 2014.05.14.

Cordier A.G., S. Guitton, C. Vauloup-Fellous, L. Grangeot-Keros, A. Benachi and O. Picone. 2012a. Awareness and knowledge of congenital cytomegalovirus infection among health care providers in France. J. Clin. Virol. 55: 158-163.

Cordier A.G., S. Guitton, C. Vauloup-Fellous, L. Grangeot-Keros, J.M. Ayoubi, A. Benachi and O. Picone. 2012b. Awareness of cytomegalovirus infection among pregnant women in France. J. Clin. Virol. 53: 332-337.

Dahle A.J., K.B. Fowler, J.D. Wright, S.B. Boppana, W.J. Britt and R.F. Pass. 2000. Longitudinal investigation of hearing disorders in children with congenital cytomegalovirus. J. Am. Acad. Audiol. 11: 283-290.

Davidson A.C. and D.V. Hinkley. 1997. Bootstrap methods and their application. $1^{\text {st }}$ ed. University Press, Cambridge.

de Ory F., R. Ramírez, L. García Comas, P. León, M.J. Sagües and J.C. Sanz. 2004. Is there a change in cytomegalovirus seroepidemiology in Spain? Eur. J. Epidemiol. 19: 85-89.

de Vries J.J., E.W. van Zwet, F.W. Dekker, A.C. Kroes, P.H. Verkerk and A.C. Vossenet. 2013. The apparent paradox of maternal seropositivity as a risk factor for congenital cytomegalovirus infection: a population-based prediction model. Rev. Med. Virol. 23: 241-249. Dollard S.C., S.D. Grosse and D.S. Ross. 2007. New estimates of the prevalence of neurological and sensory sequelae and mortality associated with congenital cytomegalovirus infection. Rev. Med. Virol. 17: 355-363.

Enders G., U. Bäder, U. Bartelt and A. Daiminger. 2003. Cytomegalovirus (CMV) prevalence and frequency of CMV primary infections in pregnant women (in German). Bundesgesundheitsblatt - Gesundheitsforschung - Gesundheitsschutz 46: 426-432.

Engman M.L., G. Malm, L. Engstrom, K. Petersson, E. Karltorp, K. Tear Fahnehjelm, I. Uhlen, C. Guthenberg and I. LewensohnFuchs. 2008. Congenital CMV infection: prevalence in newborns and the impact on hearing deficit. Scand. J. Infec. Dis. 40: 935-942. Fowler K.B., A.J. Dahle, S.B. Boppana and R.F. Pass RF. 1999. Newborn hearing screening: will children with hearing loss caused by congenital cytomegalovirus infection be missed? J. Pediatr. 135: 60-64 Gardella C. 2008. Cytomegalovirus in Pregnancy. In: The Global Library of Women's Medicine DOI 10.3843/GLOWM.10182.

Godzik P., A. Kołakowska, K. Madaliński, M. Stepień, A. Zielińnski, A. Góralewska, M. Kazimierska, R. Kunc-Kozioł, B. Nadolska, A. Pawłowska and others. 2012. Prevalence of anti-HCV antibodies among adults in Poland-results of cross-sectional study in general population (in Polish). Przegl. Epidemiol. 66: 575-580. Gratacap-Cavallier B., J.L. Bosson, P. Morand, N. Dutertre, B. Chanzy, P.S. Jouk, C. Vandekerckhove, P. Cart-Lamy and J.M. Seigneurin. 1998. Cytomegalovirus seroprevalence in French pregnant women: parity and place of birth as major predictive factors. Eur. J. Epidemiol. 14: 147-152.

Hyde T.B., D.S. Schmid and M.J. Cannon. 2010. Cytomegalovirus seroconversion rates and risk factors: implications for congenital CMV. Rev. Med. Virol. 20: 311-326.

Ikuta K., T. Minematsu, N. Inoue, T. Kubo, K. Asano, K. Ishibashi, T. Imamura, H. Nakai, T. Yoshikawa, H. Moriuchi and others. 2013. Cytomegalovirus (CMV) glycoprotein H-based serological analysis in Japanese healthy pregnant women, and in neonates with congenital CMV infection and their mothers. J. Clin. Virol. 58: 474-478.

Imbs D. and H. Rudnicka. 1987. Seroepidemiological studies for the detection of cytomegalovirus (CMV) and herpes simplex virus (HSV) infections among girls and women in Poland (in Polish). Przegl. Epidemiol. 41: 286-294.

Kenneson A. and M.J. Cannon. 2007. Review meta-analysis of the epidemiology of congenital cytomegalovirus (CMV) infection. Rev. Med. Virol. 17: 253-276.
Kiss P., D. de Bacquer, L. Sergooris, M. de Meester and M. VanHoorne. 2002. Cytomegalovirus infection: an occupational hazard to kindergarten teachers working with children aged 2.5-6 years. Int. J. Occup. Environ. Health 8: 79-86.

Knowles S.J., K. Grundy, I. Cahill, M.T. Cafferkey amd M. Geary. 2005. Low cytomegalovirus sero-prevalence in Irish pregnant women. Ir. Med. J. 98: 210-212.

Korndewal M.J., L. Mollema, I. Tcherniaeva, F. van der Klis, A.C. Kroes, A.M. Oudesluys-Murphy, A.C. Vossen and H.E. de Melker. 2015. Cytomegalovirus infection in The Netherlands: seroprevalence, risk factors, and implications. J. Clin. Virol. 63: 53-58. Liu X., J. Yuan, A.W. Wu, P.W. McGonagill, C.S. Galle and J.L. Meier. 2010. Phorbol ester-induced human cytomegalovirus major immediate-early (MIE) enhancer activation through PKCdelta, CREB, and NF-kappaB desilences MIE gene expression in quiescently infected human pluripotent NTera2 cells. J. Virol. 84: 8495-8508.

Ludwig A. and H. Hengel. 2009. Epidemiological impact and disease burden of congenital cytomegalovirus infection in Europe. Euro Surveill. 14:26-32.

Manicklal S., V.C. Emery, T. Lazzarotto, S.B. Boppana and R.K. Gupta. 2013. The "silent" global burden of congenital cytomegalovirus. Clin. Microbiol. Rev. 26: 86-102.

Mustakangas P., S. Sarna, P. Ammälä, M. Muttilainen, P. Koskela and M. Koskiniemi. 2000. Human cytomegalovirus seroprevalence in three socioeconomically different urban areas during the first trimester: a population-based cohort study. Int. J. Epidemiol. 29: $587-591$

Nyholm J.L. and M.R. Schleiss. 2010. Prevention of maternal cytomegalovirus infection: current status and future prospects. Int. J. Womens Health 9:23-35.

Price S.M., E. Bonilla, P. Zador, D.M. Levis, C.L. Kilgo and M.J. Cannon. 2014. Educating women about congenital cytomegalovirus: assessment of health education materials through a web-based survey. BMC Women's Health 14: 144

Revello M.G., G. Campanini, A. Piralla, M. Furione, E. Percivalle, M. Zavattoni and G. Gerna. 2008. Molecular epidemiology of primary human cytomegalovirus infection in pregnant women and their families. J. Med. Virol. 80: 1415-1425.

Revello M.G., E. Fabbri, M. Furione, M. Zavattoni, D. Lilleri, B. Tassis, A. Quarenghi, C. Cena, A. Arossa, L. Montanari and others. 2011. Role of prenatal diagnosis and counseling in the management of 735 pregnancies complicated by primary human cytomegalovirus infection: a 20-year experience. J. Clin. Virol. 50: 303-307.

Shkedy Z., M. Aerts, G. Molenberghs, P. Beutels and P. Van Damme. 2006. Modelling age-dependent force of infection from prevalence data using fractional polynomials. Stat. Med. 25: 1577-1591.

Sinclair J. and P. Sissons. 2006. Latency and reactivation of HCMV J. Gen. Virol. 87: 1763-1779.

Tookey P.A., A.E. Ades and C.S. Peckham. 1992. Cytomegalovirus prevalence in pregnant women: the influence of parity. Arch. Dis. Child 67: 779-783.

Wang C., X. Zhang, S. Bialek and M.J. Cannon. 2011. Attribution of congenital cytomegalovirus infection to primary versus nonprimary maternal infection. Clin. Infect. Dis. 52: 1-13.

Yamamoto A.Y., M.M. Mussi-Pinhata, S.B. Boppana, Z. Novak, V.M. Wagatsuma, P.F. Oliveira, G. Duarte and W.J. Britt. 2010 Human cytomegalovirus reinfection is associated with intrauterine transmission in a highly cytomegalovirus-immune maternal population. Am. J. Obstet. Gynecol. 202: 297e1-297e8.

Yamamoto A.Y., R.A. Castellucci, D.C. Aragon and M.M. MussiPinhata. 2013. Early high CMV seroprevalence in pregnant women from a population with a high rate of congenital infection. Epidemiol. Infect. 141: 2187-2191. 